\title{
Deviation from the Unimolecular Micelle Paradigm of PAMAM Dendrimers Induced by Strong Interligand Interactions
}

\author{
Bo Wang, ${ }^{\dagger}$ Nicholas K. Geitner, ${ }^{\ddagger}$ Thomas P. Davis, ${ }^{\S, \|}$ Pu Chun Ke, ${ }^{\S}$ David A. Ladner, ${ }^{\perp}$ and Feng Ding ${ }^{*} \dagger$ \\ ${ }^{\dagger}$ Department of Physics and Astronomy, Clemson University, Clemson, South Carolina 29634, United States \\ ${ }^{\ddagger}$ Department of Civil and Environmental Engineering, Duke University, Durham, North Carolina 27708, United States \\ ${ }^{\S}$ ARC Centre of Excellence in Convergent Bio-Nano Science and Technology, Monash Institute of Pharmaceutical Sciences, Monash \\ University, 381 Royal Parade, Parkville, VIC 3052, Australia \\ "Department of Chemistry, Warwick University, Gibbet Hill, Coventry CV4 7AL, United Kingdom \\ ${ }^{\perp}$ Department of Environmental Engineering and Earth Sciences, Clemson University, Clemson, South Carolina 29634, United States
}

Supporting Information

ABSTRACT: PAMAM (polyamidoamine) dendrimers have been recently exploited as efficient and biocompatible unimolecular micelles for oil spill remediation utilizing their robust encapsulation capability. However, experimental evidence suggested that contrasting dispersion mechanisms of PAMAM exist toward different types of hydrocarbon ligands, including linear and polyaromatic oil molecules. Specifically, the dispersion of linear hydrocarbons by PAMAM was found to violate the unimolecular micelle convention by forming molecular complexes orders of magnitude larger than a single PAMAM. It is, therefore, essential to re-examine the dispersion mechanisms of PAMAM toward different types of ligands in order to facilitate dendrimer applications in environmental remediation, catalysis, and nanomedicine. Here, we applied atomistic discrete molecular dynamics simulations to study generation-four (G4) PAMAM dendrimers dispersing hexadecane $(\mathrm{C} 16)$ and phenanthrene $(\mathrm{PN})$, two representative linear and polyaromatic hydrocarbons in crude oil. We observed a strong cooperativity in the binding of both C16 and PN to PAMAM dendrimers, especially with C16. Simulations of multiple PAMAM molecules interacting with many hydrocarbons illustrated that phenanthrene bound to individual dendrimers to render a unimolecular micelle, while multiple C16 molecules formed a large droplet enclosed and stabilized by multiple PAMAM dendrimers to assemble into a multimolecular micelle. Our analysis revealed that such deviation of the PAMAM-ligand architecture from the conventional unimolecular micelle paradigm arose from strong interligand interactions between linear hydrocarbons.

\section{INTRODUCTION}

Dendrimers are a class of synthetic, highly controlled macromolecules with a fractal-like structure. Well-defined branching units emanate from the central core, while the number of branching iterations defines the "generation" of the dendrimer. ${ }^{1}$ The central core, interior branching units, and terminal units of the dendrimer can be individually chosen, allowing for great design flexibility. ${ }^{1}$ As a result, the size, hydrophobicity, and surface functionalities of the dendrimer can be tailored for a wide variety of applications. PAMAM (polyamidoamine) dendrimers are one of the most commonly studied dendrimers. ${ }^{1-4}$ At neutral $\mathrm{pH}$, the PAMAM dendrimers are composed of a hydrophobic interior terminated by hydrophilic surface amines, which are protonated under physiological conditions to render a so-called "unimolecular micelle" stucture. ${ }^{5-11}$ Because of their unique physicochemical properties, PAMAM dendrimers have potential applications impacting many fields. In biomedicine, for example, PAMAM dendrimers can serve as carriers by either linking drug molecules to their surface or encapsulating the drugs inside their interior cavity. ${ }^{12}$ For weakly acidic drugs, the main driving forces for noncovalent binding with dendrimers are electrostatic interactions and hydrogen bonding., 33,14 For example, generation-four (G4) PAMAM dendrimers with 64 surface amines are able to encapsulate as many as 78 ibuprofen molecules. ${ }^{15}$ PAMAM dendrimers have also been used to encapsulate and deliver small hydrophobic pharmaceutical molecules for enhanced water solubility and biodistribution. ${ }^{3}$ Solvent conditions such as polarity, ionic strength, and $\mathrm{pH}$ affect the conformation of PAMAM dendrimers ${ }^{16,17}$ and thus their encapsulation efficiency, as the dendrimer maximum loading capacity is directly related to the shape, size, and conformation of the dendrimer. ${ }^{18,19}$ However, the density of dendrimer surface groups rapidly increases with generation,

Received: May 25, 2015

Revised: July 27, 2015

Published: July 28, 2015 
which may sterically block guest molecules from partitioning into the dendrimer interior. ${ }^{19}$ At generation 7 or higher, the surface groups reach the de Gennes dense packing limit to seal the interior.

In addition to drug delivery, the hosting capability of dendrimers can also be utilized in environmental applications such as water purification, desalination, and pollution remediation. $^{20-22}$ Commercially available oil dispersants including Corexit have been in use for treating oil spills worldwide. $^{23,24}$ However, the potential toxicity of oil dispersants has recently become a great concern, particularly after their large-scale deployment in the 2010 Deepwater Horizon Spill. ${ }^{24-27}$ It is, therefore, imperative to identify an alternative oil dispersant with high biocompatibility while still maintaining a relatively high efficiency. We have previously proposed and verified in lab-scale studies that cationic PAMAM dendrimers can act as efficient oil dispersants ${ }^{20}$ for both polyaromatic and linear hydrocarbons, two key components of crude oil. In our previous experiment, linear hexadecane (C16) and polyaromatic phenanthrene (PN) were used as model hydrocarbons, both of which were hydrophobic while their size difference was negligible relative to that of a G4-PAMAM dendrimer. When the model hydrocarbons were associated with the dendrimers in water, the average hydrodynamic sizes of the aggregates were significantly different: $200 \mathrm{~nm}$ for G4-C16 and $9 \mathrm{~nm}$ for G4-PN complexes, respectively, indicating that PAMAM dendrimers dispersed linear and aromatic hydrocarbon through drastically different mechanisms. ${ }^{20}$ Specifically, the hydrodynamic radius of G4-PN was comparable to that of the G4 dendrimer, ${ }^{20}$ suggesting a single PAMAM dendrimer encapsulating multiple $\mathrm{PN}$ molecules while retaining a unimolecular micelle structure. In contrast, the size of G4C16 was 2 orders of magnitude larger than that of a single G4PAMAM, indicating that a large multiple-molecular complex was formed. It was hypothesized that, when a linear C16 molecule was encapsulated, there remained one end protruding out of the G4 molecule. ${ }^{20}$ This enabled bridging with surrounding G4 molecules to create the complexes. ${ }^{20}$ However, the experimental data were insufficient to clearly validate the hypothesis, so the structure of these supramolecular complexes and the mechanism for such clear deviation from PAMAM acting as a unimolecular micelle in ligand encapsulation remain unknown.

There have been numerous studies including both experiments and computations on the mechanisms of dendrimers encapsulating single target molecules. ${ }^{2,3,12}$ Electrostatic interactions, hydrophobic effects, or hydrogen bonding typically account for the formation of host-guest structures. ${ }^{3,13-15}$ Almost ubiquitously, the structures are described as dendrimer hosts with molecular guests forming unimolecullar micelles that do not bind with surrounding micelles; however, as mentioned above, experimental evidence with PAMAM dendrimers and C16 suggests that the formation of a supramolecular complex containing multiple dendrimers and ligands could occur. ${ }^{20}$

To elucidate the molecular mechanisms of dendrimers as dispersants, computational modeling has been applied to bridge the gap between experimental observations and actual molecular systems. ${ }^{18}$ Because of computational limitations, interactions of ligands with single dendrimers were usually modeled previously. Recently, in the absence of ligands, intermolecular interaction between two PAMAM dendrimers has been studied using umbrella sampling molecular dynamics (MD), where effective repulsion between cationic PAMAM and weak attraction between neutrally charged PAMAM were found. ${ }^{28}$ We hypothesize that, in addition to the dendrimerligand interactions, ligand-ligand interactions are important to overcome the repulsion between cationic PAMAM dendrimers in forming the supramolecular complexes observed experimentally. ${ }^{20}$ Hence, in order to fully understand the mechanisms governing dendrimer's function as an oil dispersant as well as a host for catalysts and drug molecules, it is necessary to examine both dendrimer-ligand and ligandligand interactions.

Here, we applied atomistic discrete molecular dynamics (DMD) simulations to study the dispersion mechanisms of G4PAMAM dendrimers with C16 and PN. DMD is a special type of molecular dynamics algorithm, which features rapid sampling efficiency, and has been recently shown to recapitulate the structures and dynamics of PAMAM dendrimers of different generations, surface groups, and $\mathrm{pH}$ conditions. ${ }^{18}$ We systematically studied the binding of a single PAMAM dendrimer with single and multiple ligands, as well as two PAMAM dendrimers interacting with multiple ligands. From DMD simulations, we found that both $\mathrm{C} 16$ and PN displayed a high cooperativity in binding dendrimers. Differences in structural, dynamic, and energetic properties between these two types of hydrocarbons led to drastically different clustering behaviors in the absence and presence of dendrimers. Compared to the rigid PN molecule, strong interligand interaction between the flexible C16 resulted in weaker solubility, i.e., the association and condensation of the ligands in water. We found that the same physicochemical properties also led to the formation of multidendrimer micelles in the presence of PAMAM dendrimers, thereby revising the unimolecular micelle paradigm of PAMAM dendrimer as a host agent. As drug molecules usually possess hydrophobic and aromatic moieties, the results of this study may be extended beyond the scope of environmental remediation to studies of dendrimers for drug delivery, catalysis, and biosensing.

\section{METHODS}

Discrete Molecular Dynamics Simulation. Discrete molecular dynamics (DMD) is a special type of molecular dynamics algorithm where interatomic interactions are modeled by square-well potential functions instead of continuous potentials. A more detailed description of the DMD algorithm can be found elsewhere. ${ }^{29,30}$ DMD has been proved to be a powerful method in simulating biomacromolecules such as proteins. ${ }^{31-33}$ Recently, we have successfully applied DMD in simulating dendrimers with various structures. ${ }^{18}$ We used a united-atom representation for the dendrimer-hydrocarbon systems in which all heavy atoms and polar hydrogens were explicitly modeled. Interatomic interactions including van der Waals (VDW), solvation, electrostatic, and hydrogen bond interactions were modeled by a physical force field adapted from Medusa. ${ }^{34,35}$ Bond length, angle, and dihedrals were taken from CHARMM 19. ${ }^{36}$ The solvation energy was included using the Lazaridis-Karplus implicit solvent model, EEF1. ${ }^{37}$ The distance- and angular-dependent hydrogen bond interaction was modeled using a reaction-like algorithm. ${ }^{34} \mathrm{We}$ used the Debye-Hückel approximation to model the screened electrostatic interactions between charged atoms. The Debye length was approximately $1 \mathrm{~nm}$ by assuming a water relative permittivity of 80 , and a monovalent electrolyte concentration of $0.1 \mathrm{M}$. 
The periodic boundary condition was used in DMD simulations. We adopted the Anderson's thermostat to perform constant temperature simulations. In DMD simulations, the temperature has the unit of $\mathrm{kcal} /\left(\mathrm{mol} \cdot k_{B}\right)$, where $k_{B}$ is the Boltzmann constant. Our simulations were conducted across a temperature range of $0.50-0.80 \mathrm{kcal} /\left(\mathrm{mol} \cdot k_{B}\right)$, corresponding to $250-400 \mathrm{~K}$ with a scaling factor of $\sim 500$. We note that our implicit solvent approach could not capture the temperature dependence of the hydrophobic interactions mediated by water molecules. On the other hand, we expect that the extrapolated enthalpy and entropy values from DMD simulations at a wide range of temperatures were good approximations near the room temperature where the parameters of implicit solvent model were tabulated. ${ }^{37}$ At each temperature, energy minimization was first carried out for 1000 time steps (approximately $0.05 \mathrm{~ns}$ ).

PAMAM Dendrimer Model. Generation-four poly(amidoamine) (PAMAM) dendrimers are highly positively charged polymers composed of a central core, branching units, and 64 amine surface groups. To emulate a solution $\mathrm{pH}$ of 8.2, all the amine groups of dendrimers are fully protonated while all interior amine groups remained deprotonated. Because of the high number charges of PAMAM dendrimer molecules, the local salt concentration near PAMAM is higher than that of the bulk, and consequently, the screening effect near the surface is stronger than the bulk. It has been suggested ${ }^{38}$ that, in highly charged systems, Debye-Hückel approximation with neutralizing counterions can be used to account for the increased screening effect. Therefore, the net charges of the molecular systems were maintained at zero by adding offsetting counterions $(\mathrm{Cl}-)$. We computed the radial density distribution of charged atoms, including $\mathrm{Cl}^{-}$ions and primary amines $\left(-\mathrm{NH}_{3}{ }^{+}\right.$) (Figure $\mathrm{S} 2$ ). We found that the counterions (Cl-) indeed mostly stayed near the protonated primary amines as expected. In each simulation, energy minimization was first performed, followed by further equilibration. In equilibrium simulations, simulations with two dendrimers were carried out through 0.5 million time steps $(\sim 25 \mathrm{~ns})$ while all others were carried out for 1 million steps $(\sim 50 \mathrm{~ns})$, all of which corresponded to an average of approximately $72 \mathrm{CPU}$ hours.

Simulations of One Dendrimer with One Ligand System. For each type of ligand, we performed DMD simulations at different temperatures (from 250 to $400 \mathrm{~K}$ ). At each temperature, we performed 20 independent simulations with different initial configurations, where hydrocarbons were positioned with random orientations and distances to the dendrimer.

Umbrella Sampling of One Dendrimer with One C16. The intermolecular distance between a central carbon of the PAMAM core and the center atom of C16 (i.e., the 8th carbon), $r_{C}$, was chosen as the reaction coordinate. We performed 10 replicas of DMD simulations, each of which had an infinite square-well bias potential as a function of $r_{C}$. The upper and lower bounds of the biased potentials were assigned to overlap with each other: $\{0,10.5\},\{9.5,14.5\}$, $\{13.5,18.5\},\{17.5,22.5\},\{21.5,26.5\},\{25.5,30.5\},\{29.5$, $34.5\},\{33.5,38.5\},\{37.5,42.5\}$, and $\{41.5,137.5\} \AA$. The first and last bias potentials had wider ranges because the ligand was either completely bound or unbound, correspondingly. All simulations were carried out for 1 million steps ( $\sim 50 \mathrm{~ns})$ at $T=$ $300 \mathrm{~K}$. We utilized 5000 snapshots evenly distributed throughout the final $25 \mathrm{~ns}$ of the simulations to compute the intermolecular distances, $r_{C}$. The weighted histogram analysis method (WHAM $)^{39}$ was applied to estimate the one-dimensional (1D) potential of mean force (PMF) with respect to the intermolecular distance $r_{C}$.

Simulations of One Dendrimer with 20 Ligands. Twenty ligands (20 C16 or $20 \mathrm{PN}$ ) were first assigned with random orientations and distances to the dendrimer. Then, we let the system run at the lowest simulation temperature $(T=$ $250 \mathrm{~K}$ ) until equilibrium was reached (2 million steps, approximately $100 \mathrm{~ns}$ ). At this temperature, all ligands were eventually encapsulated by the dendrimer. Then, we chose snapshots of the equilibrium states as our starting state for the dissociation simulations. The dissociation simulations (as well as all the following simulations) were performed at the same temperature range $(250-400 \mathrm{~K})$. At each temperature, we performed 10 independent simulations with different initial velocities assigned according to the Maxwell-Boltzmann distribution.

Simulations of Hydrocarbon Clusters. We followed the same protocol as above where $20,40,60$, or 80 ligands (C16 or $\mathrm{PN}$ ) were first assigned with random orientations and distances to each other. Then, we let the system run at $T=250 \mathrm{~K}$ until equilibrium was reached ( 1 million steps, approximately $50 \mathrm{~ns}$ ). Those hydrophobic ligands assembled quickly to become a single cluster. Ten snapshots of hydrocarbon clusters along the association simulations were chosen as the initial structures for the dissociation simulations.

Preparation of the Two Dendrimers Binding with $\mathbf{8 0}$ Ligands. Two dendrimers were first positioned away from the 80 -ligand cluster (obtained from the previous simulation). Then, we let the system run at $T=300 \mathrm{~K}$ for 0.5 million steps, approximately $25 \mathrm{~ns}$. Two dendrimers were bound to the cluster eventually. To initialize the structures for the following dissociation process, we repeated the above approaching process at lower temperature $(T=250 \mathrm{~K})$ and 10 final snapshots of the simulation were chosen separately so that simulations could start from 10 different configurations. The dissociation processes were performed for another 0.5 million steps until an equilibrium of the system was reached.

Contact Definition and the Clustering Analysis. A contact between two molecules was defined when at least one pair of heavy atoms from each molecule is within $5.0 \AA$. We used the single-linkage criterion to define a molecular cluster, where a molecule belonged to an existing cluster if it made any contacts with members of the cluster. The size of a cluster is defined as the number of member molecules. The mean and standard deviation of contacts and cluster size at each temperature were obtained from system snapshots in equilibrium. In the case of two G4 dendrimers with multiple hydrocarbons, we collected 1000 snapshots evenly distributed along the final $5 \mathrm{~ns}$ of the simulations. In all other simulations, we utilized 5000 snapshots evenly distributed throughout the final $25 \mathrm{~ns}$ of the simulations.

Structural Analysis of PAMAM Dendrimer. We used two structural parameters to capture the structure information on the dendrimer, including radius of gyration, $R g$, to quantify dendrimer size, and ellipticity, $e$, to quantify the shape of the dendrimer. Both $R g$ and ellipticity can be obtained from the momentum of inertia tensor, $I$ (diazotization of $3 \times 3$ matrix results into three eigenvalues, $I_{x}<I_{y}<I_{z}$ ):

$$
R g=\sqrt{\left(I_{x}+I_{y}+I_{z}\right) / m}
$$



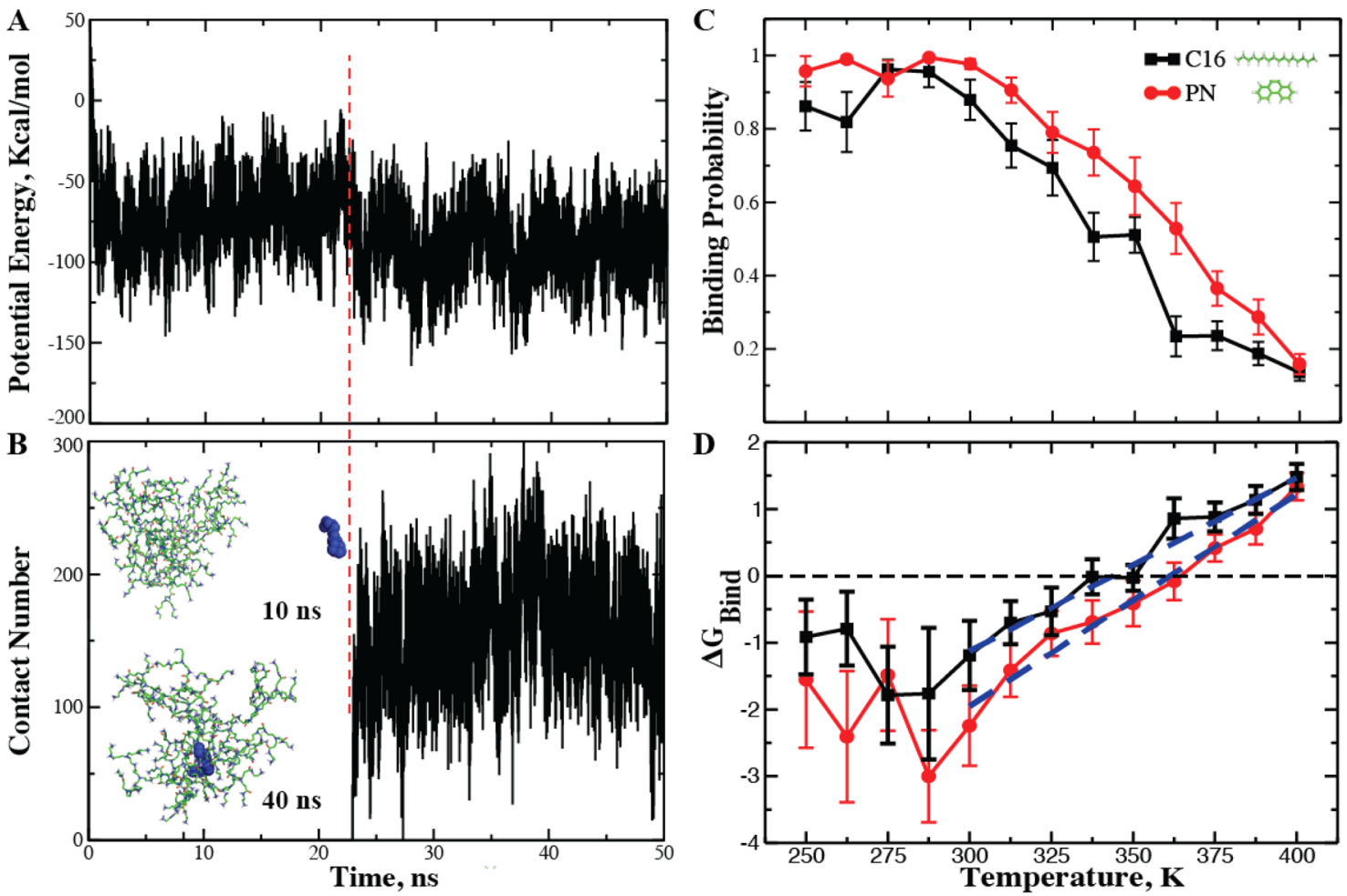

Figure 1. Binding simulations of one hydrocarbon ligand interacting with a single dendrimer. (A) The total potential energy and (B) the number of intermolecular contacts were plotted as functions of simulation time from a typical DMD simulation of one dendrimer interacting with one C16 ligand at $T=300 \mathrm{~K}$. Two snapshots at 10 and $40 \mathrm{~ns}$ are shown as the insets in panel B. The vertical dashed line corresponds to the time when two molecules bound to each other. (C) The binding probabilities, $P_{\text {bind }}$, of $\mathrm{C} 16$ and $\mathrm{PN}$ with dendrimer were computed as functions of temperature. (D) The binding free energy, $\Delta G_{b i n d}$ of C16 and PN with dendrimer was estimated using $P_{\text {bind. }}$. The blue dashed lines in (D) correspond to linear fits from $T=300 \mathrm{~K}$ to $T=400 \mathrm{~K}$. The slope and intercept of the fitting line correspond to $\Delta S$ and $\Delta H$, respectively.

$$
e=\sqrt{1-\left(I_{x} / I_{z}\right)}
$$

Here, $m$ is the total mass of the dendrimer.

\section{RESULTS AND DISCUSSION}

We used united-atom representations to model both PAMAM and hydrocarbon molecules in our atomistic DMD study (Methods section). We modeled interatomic interactions with van der Waals, solvation, electrostatic, and hydrogen bonds. We used the EEF1 implicit solvent model ${ }^{40}$ in $\mathrm{CHARMM}^{37}$ to estimate the solvation energy. To ensure sufficient sampling, multiple independent DMD simulations for each molecular system-e.g., one ligand with one dendrimer, multiple ligands with one dendrimer, multiple ligands with multiple dendrimers, as well as multiple ligands alone in solution-were performed with different initial conditions.

Aromatic PN Has a Stronger Binding to a Single G4 PAMAM Dendrimer than Linear C16. We first studied the simple case of a single cationic G4 PAMAM dendrimer interacting with a single hydrocarbon, including C16 and PN. For each type of ligand, we performed DMD simulations at different temperatures to study the binding thermodynamics. At each temperature, we performed 20 independent simulations with different initial configurations, where hydrocarbons were positioned with random orientations and distances to the dendrimer and each simulation lasted $50 \mathrm{~ns}$ (Methods section). In DMD simulations, a ligand was able to bind the dendrimer after random diffusion (Figure 1A). Because of the small size of a single ligand, the energy difference between the bound and the unbound state was small compared to fluctuations. We computed the number of intermolecular atomic contacts between ligand and dendrimer to characterize their binding (Figure 1B). The two molecules were counted as bound if they made any intermolecular contacts between heavy atoms. For each type of hydrocarbon ligand, we calculated its binding probability, $P_{b i n d}$, to the dendrimer as a function of temperature (Figure 1C). We used the final $25 \mathrm{~ns}$ of each simulation and calculated the averages and standard deviations over 20 replicates. At low temperatures, the hydrocarbons stayed bound with $P_{b i n d} \sim 1$ and the ligands were encapsulated inside the dendrimer. As the temperature increased, $P_{b i n d}$ decreased due to thermal fluctuations. Assuming a canonical ensemble with the bound and unbound states, we can estimate the binding free energy, $\Delta G_{b i n d}=-k_{B} T \ln P_{b i n d} /\left(1-P_{b i n d}\right)$. For each temperature, we obtained the $\Delta G_{b i n d}$ of $\mathrm{C} 16$ and PN (Figure 1D). To verify our above approach of $\Delta G_{b i n d}$ estimation, we performed umbrella sampling to study the binding of a C16 with a single dendrimer at $T=300 \mathrm{~K}$ (Methods section). We estimated the PMF as a function of the intermolecular distance, $r$, using WHAM $^{39}$ (Figure S3). The 1D PMF had two minima, the one with a shorter $r$ corresponding to the bound state and the other corresponding to the unbound state. The PMF barrier related to the dissociation between $\mathrm{C} 16$ and the G4 PAMAM dendrimer was smaller compared to previous computational results between polar drugs and a G5 PAMAM dendrimer, where the binding was governed by strong intermolecular hydrogen bond and electrostatic interactions. ${ }^{41}$ A lower density of amidoamine monomers in a G4 PAMAM than a G5 dendrimer may also contribute to the smaller PMF barrier. The binding free energy, estimated as the PMF 
difference between two minima, was $\sim-1.56 \mathrm{kcal} / \mathrm{mol}$, in agreement with the estimation from our unbiased simulations $\sim-1.19 \pm 0.52 \mathrm{kcal} / \mathrm{mol}$. The discrepancy was due to the fact that the actual binding free energy corresponds to the integral of the 1D PMF

$$
k_{B} T \ln \int_{d^{\ddagger}}^{\infty} \exp [-\beta P M F(r)] d r-k_{B} T \ln \int_{0}^{d^{\neq}} \exp [-\beta P M F(r)] d r
$$

where distance $d^{\neq}$corresponds to the free energy barrier separating bound and unbound states in the PMF plot, and $\beta$ denotes $1 / k_{B} T$ (Figure S3). Since the unbound basin was wider compared to the bound basin, the difference of PMF between bound and unbound basins was an overestimation. Therefore, the comparison with umbrella sampling validated our approach to estimate $\Delta G_{\text {bind }}$ from unbiased DMD simulations.

The estimated $\Delta G_{b i n d}$ has linear temperature dependence for a wide range of temperatures in simulations, and the deviation at low temperatures is probably due to the insufficient sampling of binding/unbinding events. On the basis of the temperature dependence of binding free energy, we can estimate the changes of enthalpy $(\Delta H)$ and entropy $(\Delta S)$ associated with ligand-PAMAM binding, $\Delta G_{\text {bind }}=\Delta H-T \Delta S$. Using linear regression, we estimated $\Delta H$ and $\Delta S$ for both single $\mathrm{C} 16$ and PN binding to a single PAMAM (Table 1). Compared to C16,

Table 1. Enthalpy and Entropy Changes for Various Dendrimer-Ligand Systems ${ }^{a}$

\begin{tabular}{cccc}
\multicolumn{2}{c}{ ligands } & $\Delta H, \mathrm{kcal} / \mathrm{mol}$ & $\Delta S, \mathrm{kcal} /\left(\mathrm{mol}^{*}\right.$ Kelvin $)$ \\
$\mathrm{x} 1$ & $\mathrm{C} 16$ & $-9.08(0.61)$ & $-0.0265(0.0017)$ \\
& $\mathrm{PN}$ & $-11.48(0.67)$ & $-0.0317(0.0019)$ \\
$\mathrm{*} 20$ & $\mathrm{C} 16$ & $-14.85(3.61)$ & $-0.0326(0.0096)$ \\
& $\mathrm{PN}$ & $-11.47(0.51)$ & $-0.0306(0.0014)$
\end{tabular}

${ }^{a_{T}}$ The values were obtained by fitting $\Delta G_{\text {bind }}$ as a function of temperature (standard error of linear regression in bracket). The fitting temperature range was from 300 to $400 \mathrm{~K}$, except in the case of $\mathrm{C} 16 \times 20$ (from $350 \mathrm{~K}$ ).

PN had a stronger gain of enthalpy but also a higher loss of entropy upon binding PAMAM. A higher $\Delta H$ for PN was due to its planar, aromatic structure, which had stronger van der Waals interactions with the PAMAM dendrimer than the linear C16. Also, because of the rigid planar geometry of $\mathrm{PN}$, binding with PAMAM had a stronger confinement effect than the flexible $\mathrm{C} 16$, which resulted in a higher entropy loss $\Delta S$ for PN upon binding with PAMAM.

C16 Has a Higher Binding Cooperativity to a PAMAM Dendrimer than PN. To evaluate the effect of interligand interactions on hydrocarbons binding to a dendrimer, which arises from a large number of ligands encapsulated within a dendrimer, we performed DMD simulations of $20 \mathrm{C} 16$ or PN molecules interacting with one dendrimer. As in the above single hydrocarbon study, we were interested in the average binding probability $P_{\text {bind }}$ of each ligand. In principle, if equilibrium is reached in DMD simulations, the calculated $P_{\text {bind }}$ should be independent of the initial condition, e.g., all hydrocarbon ligands initially positioned outside or encapsulated inside the dendrimer. As a simple test, we performed control association simulations starting with all C16 outside of the dendrimer and found that the results were consistent with the dissociation simulations starting from all ligands encapsulated by the dendrimer, with the only difference being that the association simulations took more time to reach equilibrium in the former configuration (Figure 2A). Therefore, in each molecular system, we initially placed all hydrocarbons encapsulated inside the dendrimer and performed DMD simulations to equilibrate the system. At each temperature, we computed the average number of ligands bound to the dendrimer (Figure 2B). We noted that the number of bound ligands per PAMAM in DMD simulations was higher than that reported in previous experiments. ${ }^{18}$ The difference between simulations and experiments is possibly because of the fact that temperatures in simulations cannot exactly correspond to the experimental temperatures and that the experimental value was estimated as a lower limit. We computed the average binding probability $P_{b i n d}$ as a function of temperature by normalizing with the total number of ligands added to the system (Figure S1). Compared to the results of a single ligand binding to the dendrimer, the increased binding probablitiy, $P_{\text {bound }}$ implied a cooperative effect for both $\mathrm{C} 16$ and PN. Following the same strategy of single ligand binding, we calculated the effective binding free energy $\Delta G_{\text {bind }}$ for each ligand (Figure 2C). We also estimated $\Delta S$ and $\Delta H$ (Table 1 ). In the case of PN, $\Delta H$ and $\Delta S$ remained approximately identical for single and multiple ligands, but the interactions among the PN ligands did decrease the system energy, leading to a slightly stronger $\Delta G_{b i n d}$ over a wide range of temperatures, suggesting a weak cooperativity. When the system had multiple $\mathrm{C} 16$ molecules, cooperative binding was so strong that we only observed partial dissociations at high temperatures (Figure 2). Fitted with only a few temperature points, the estimated $\Delta H$ and $\Delta S$ featured larger standard errors than other cases. Nevertheless, compared to single $\mathrm{C} 16$, we observed a significant increase in enthalpy gain $\Delta H$ for multiple ligand binding, while changes in $\Delta S$ are within error bars. The significant decrease in $\Delta G_{b i n d}$ for multiple $\mathrm{C} 16$ binding suggests a strong binding cooperativity. Such cooperativity can be explained by the increased hydrophobicity of the dendrimer core after binding to hydrocarbons, which, in turn, effectively enhanced the binding of additional hydrophobic ligands (Figure 2D,E).

Taken together, although PN showed higher binding affinity than $\mathrm{C} 16$ in the case of a single dendrimer interacting with one ligand, as more ligands were added to the simulation system, the relative binding strength of those two ligands reversed (Figure 2C,D). For PN, the binding free energy $\Delta G_{b i n d}$ as well as $\Delta H$ and $\Delta S$ were strikingly similar in both the one and the multiple ligand binding simulations. However, for C16, $\Delta G_{\text {bind }}$ and $\Delta H$ were significantly increased in the case of multiple ligand binding. So, what was the origin for the much stronger binding cooperativity of C16 than PN (Figure 2)?

C16 Features Strong Interligand Interaction. We next determined whether the cooperativity of hydrocarbons encapsulated by a dendrimer was due to the intrinsic physicochemical properties of each hydrocarbon species. For both $\mathrm{C} 16$ and PN, we performed DMD simulations to evaluate the association dynamics of ligands alone. As described above, we performed dissociation studies in DMD simulations where we generated hydrocarbon clusters with different sizes (i.e., comprising 20,40,60, and $80 \mathrm{C} 16$ or PN molecules) as initial states and observed the dynamics of dissociation at different temperatures (Methods section). At low temperatures, the initial clusters were stable during the course of the simulations. As the temperature increased, the size of initial clusters shrank by releasing surface hydrocarbons due to thermal fluctuations. Therefore, we computed the average size of the largest cluster 


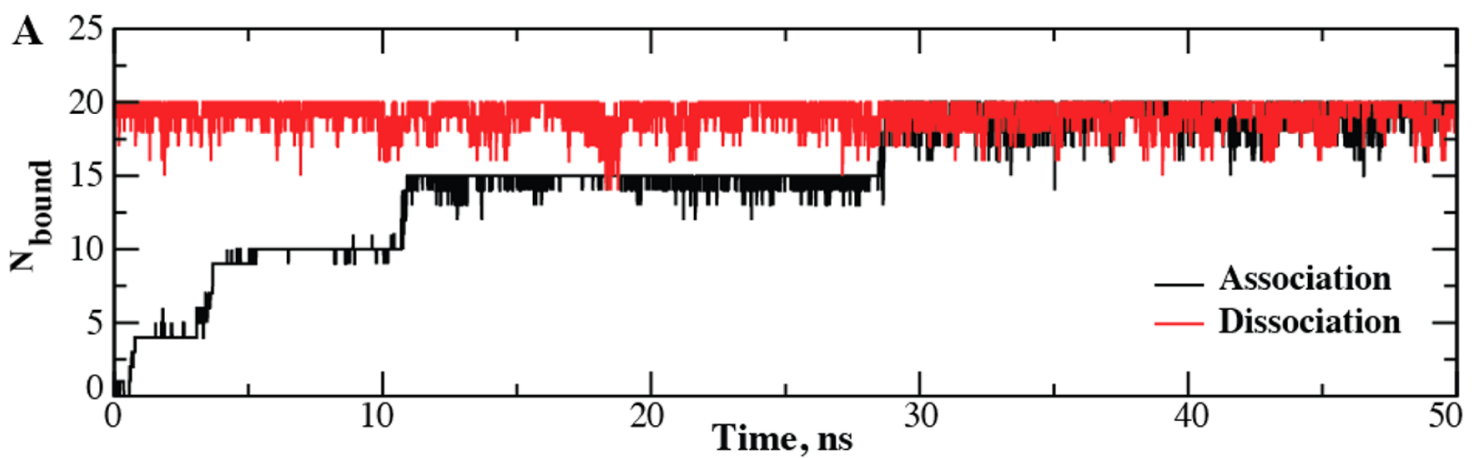

B

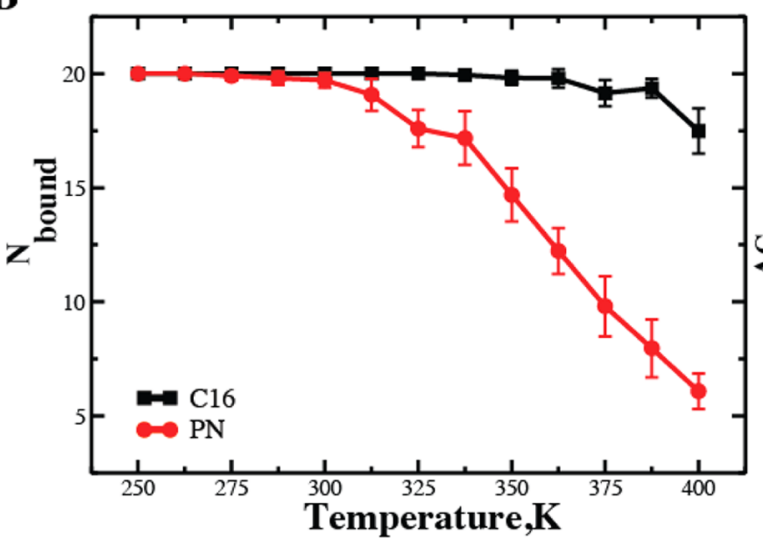

$\mathbf{D}$

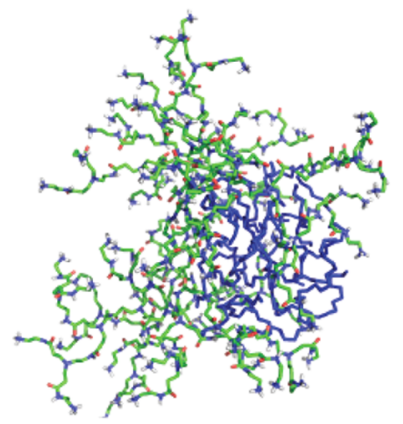

$\mathbf{E}$

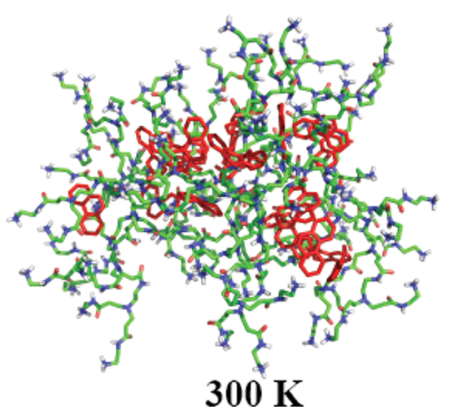

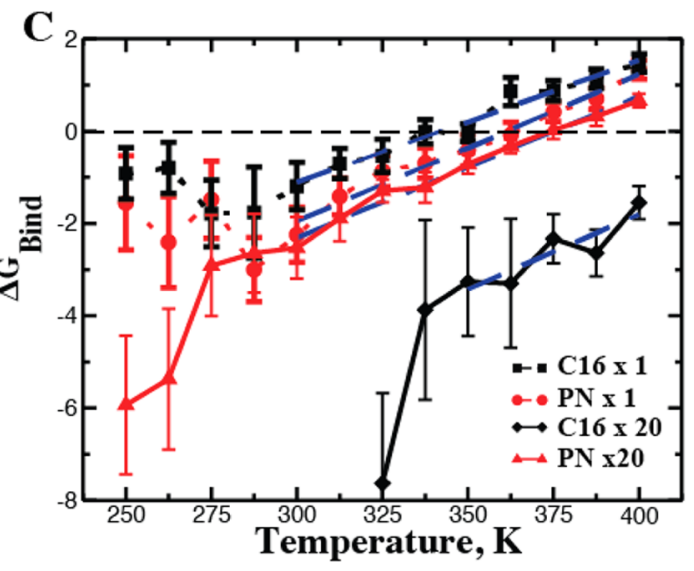

r

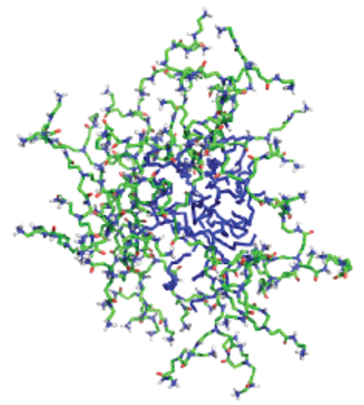

$\infty$

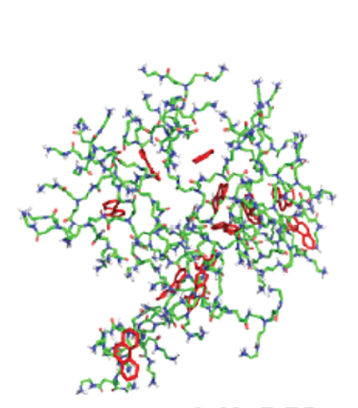

$362.5 \mathrm{~K}$

Figure 2. Simulations of 20 hydrocarbons interacting with a single dendrimer. (A) The number of C16 ligands bound to the dendrimer, $N_{b o u n d}$ as a function of simulation time was computed from both association (black line) and dissociation (red) simulations at $T=300 \mathrm{~K}$. It took approximately $30 \mathrm{~ns}$ for the association process to reach equilibrium. (B) Averaged over independent simulations at each temperature, the average $N_{\text {bound }}$ was computed for both C16 and PN. (C) The binding free energy change per ligand, $\Delta G_{b i n d}$, was computed from the normalized binding probability (Figure S1). For comparison, $\Delta G_{b i n d}$ for single hydrocarbon binding simulations was also included. The blue dashed lines correspond to linear fits from $T=300 \mathrm{~K}$ to $T=400 \mathrm{~K}$ (except for $\mathrm{C} 16 \times 20$ simulations which started from $T=350 \mathrm{~K}$ ). Snapshot structures at $T=300$ and $362.5 \mathrm{~K}$ were taken from DMD simulations for both $\mathrm{C} 16$ (D) and PN (E).

in simulations as a function of temperature (Figure $3 \mathrm{~A}$ ). A hydrocarbon ligand with stronger interligand interaction is expected to have greater thermostability, preserving its initial cluster. We found that the size of $\mathrm{C} 16$ clusters did not change significantly within the range of simulated temperatures. In contrast, PN clusters were less stable and all sizes of the 


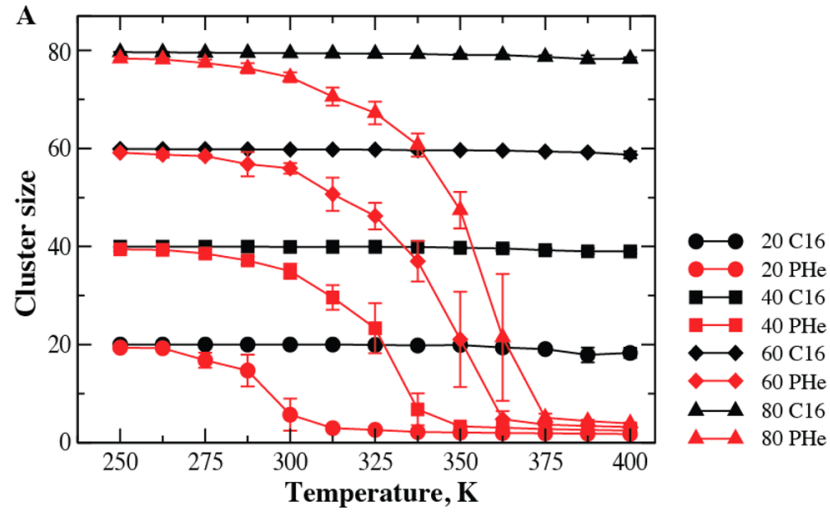

B
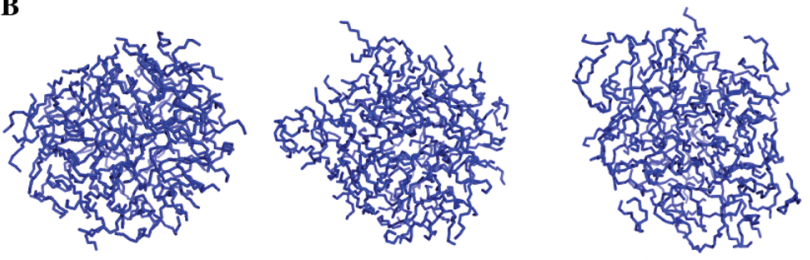

C

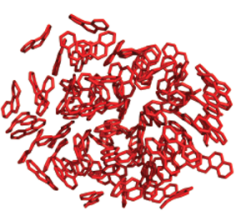

$250 \mathrm{~K}$

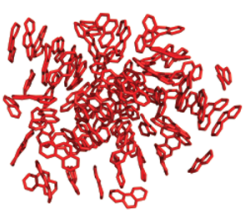

$300 \mathrm{~K}$

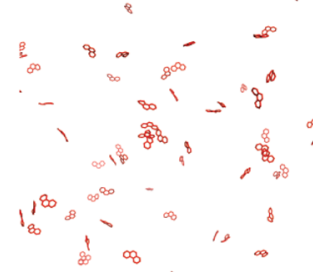

$400 \mathrm{~K}$
Figure 3. Thermostability of hydrocarbon clusters. (A) The size of hydrocarbon clusters as a function of simulation temperature. For both C16 and PN, DMD simulations of hydrocarbon clusters with initial sizes of 20,40, 60, and 80 ligands were performed at different temperatures. The number of ligands in the largest cluster was calculated to quantify the corresponding thermostability. Snapshots of structures at temperatures $T=250,300$, and $400 \mathrm{~K}$ were taken from DMD simulations for both $\mathrm{C} 16$ (B) and PN (C). The systems were in equilibrium at each sampling temperature.

simulated clusters broke into many smaller clusters at high temperatures. Since our simulations modeled the association of hydrocarbons in water implicitly, our results are consistent with the observation that PN has higher solubility in water than that of $\mathrm{C} 16 .^{18}$

The linear C16 molecule is flexible, whereas the planar aromatic PN molecule is rigid. Examination of the snapshots at different temperatures (Figure $3 \mathrm{~B}$ ) revealed that $\mathrm{C} 16$ molecules in their clusters were mostly unstructured, and thus, $\mathrm{C} 16$ clusters were liquid-like. On the other hand, $\mathrm{PN}$ had a preferred aggregation orientation, i.e., $\pi-\pi$ stacking due to their planar aromatic structure. Compared to the solid-like PN clusters, the higher entropy of $\mathrm{C} 16$ clusters contributed to their higher thermostability. Taken together, C16 featured stronger interligand interactions than $\mathrm{PN}$, which resulted in their higher binding cooperativity to PAMAM.

Strong Interligand Interactions Give Rise to the Deviation from the Unimolecular Micelle Paradigm of PAMAM Dendrimer. To test whether strong interligand and ligand-PAMAM interactions were able to overcome the repulsion between cationic PAMAM to form large molecular complexes, we next modeled the binding of multiple hydrocarbons interacting with two G4 dendrimers simultaneously. We first generated a large hydrocarbon cluster, consisting of 80
C16 (or PN) to mimic an oil droplet. Two dendrimers were initially positioned away from the oil droplet. From the above discussion, we noted that the 80 -hydrocarbon system was quite stable below $T=300 \mathrm{~K}$. We performed association simulations at $T=300 \mathrm{~K}$. We monitored both $R g$ and ellipticity (Methods section) to characterize the dynamic properties of dendrimers (Figure 4A,B). As a control, we performed DMD simulations of

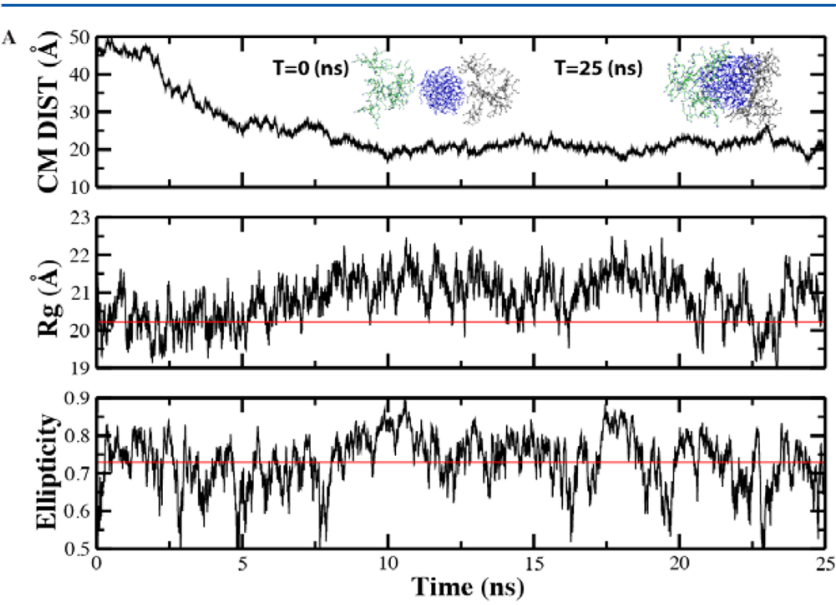

B
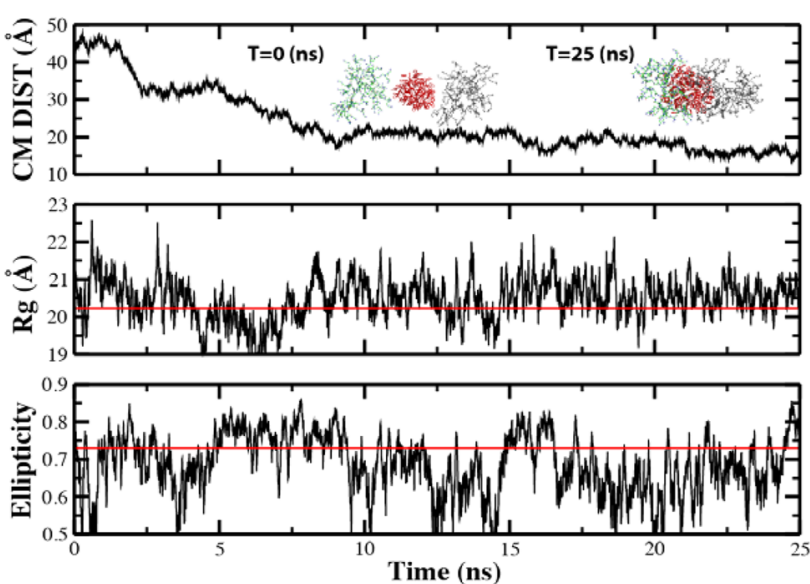

Figure 4. Association simulations of droplet binding with two dendrimers. CM distance, radii of gyration, and ellipticity of (A) dendrimer-C16 and (B) dendrimer-PN association. The red lines denote corresponding values of the G4-PAMAM dendrimer alone. Snapshot structures at the beginning ( $0 \mathrm{~ns})$ and ending $(25 \mathrm{~ns})$ are shown as insets.

the G4 PAMAM dendrimer alone at the same temperature and computed $R g$ and ellipticity (see Table S1, where we also compared our results by comparing them to the previous experimental and computational studies). The decrease of center-of-mass (CM) distances between the oil droplet and the dendrimer indicated the association. Compared to the isolated PAMAM alone, binding with the hydrocarbon droplet resulted in increased $R g$ due to conformational changes of the soft polymer nanoparticle. The final $\mathrm{CM}$ distance was smaller for PN (15 A) compared to C16 (20 A) because of the relatively smaller size of the PN droplet. The size difference of the droplet can also lead to differences in $R g$ and ellipticity of dendrimers upon droplet binding. When attached to a larger droplet like $\mathrm{C} 16$, dendrimers tended to undergo a larger conformational change in order to maximize their contact with the droplet, losing structural symmetry in the process with overall larger ellipticity. 
Starting from the complex structures, we performed dissociation simulations at different temperatures (Methods section). We computed the number of hydrocarbon ligands, $N_{L}$, each dendrimer encapsulated (or was in contact with) at equilibrium as a function of temperature (Figure 5A). $N_{L}$

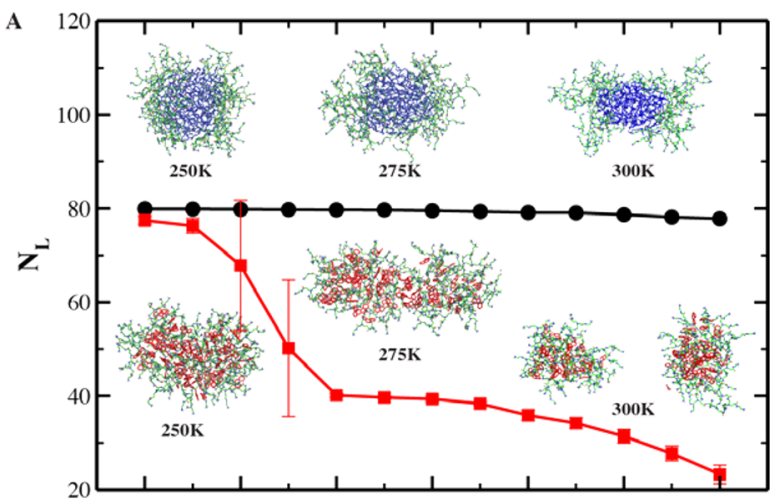

B
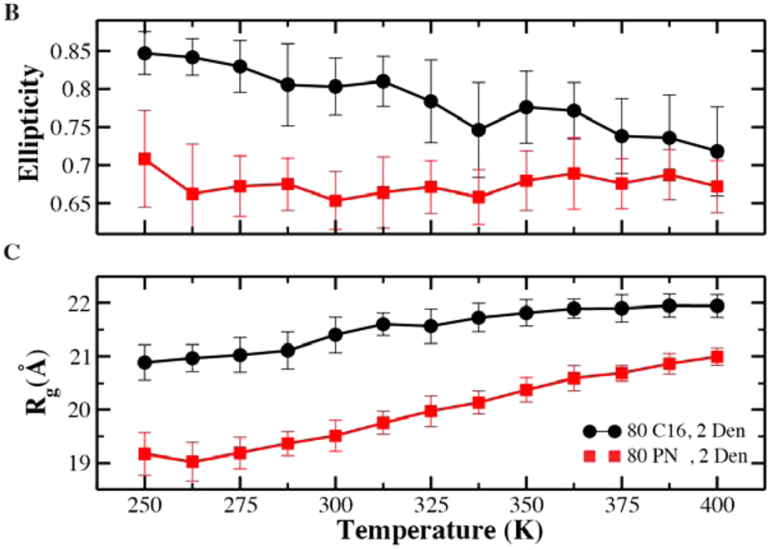

Figure 5. Simulations of multiple hydrocarbons interacting with two dendrimers. (A) The number of hydrocarbon ligands, $N_{L}$, each dendrimer encapsulated (or was in contact with) as a function of temperature. The snapshot structures in the inset were taken from DMD simulations at $T=250,275$, and $300 \mathrm{~K}$ for both $\mathrm{C} 16$ and PN. (B) Ellipticity and (C) radius of gyration illustrated the contrasting structures of PAMAM while binding to C16 and PN. The systems were in equilibrium at each sampling temperature.

corresponded to 80 if both dendrimers bound to the initial cluster (e.g., inset snapshot at $T=250 \mathrm{~K}$ in Figure 5A). The number drastically reduced if the two dendrimers did not bind to the same cluster, reflecting the average of cluster sizes bound to each dendrimer. For C16, we can see the $N_{L}$ value was approximately 80 across the entire temperature range, suggesting that both dendrimers were bound to the initial hydrocarbon cluster without observed dissociation. The branching units of dendrimers formed multiple hydrophobic interactions with the cluster, rendering the binding very robust. In contrast, we observed a completely different behavior for the PN cluster interacting with two dendrimers. As the temperature increased, the average loading for each dendrimer decreased. On the basis of the snapshot structure (e.g., $T=250 \mathrm{~K}, 270 \mathrm{~K}$ ), we noted that the cluster became thinner with increasing temperature and eventually split $(T=300 \mathrm{~K})$. At temperatures higher than $300 \mathrm{~K}$, the average $N_{L}$ became approximately a half of the initial cluster size, $\sim 40$, suggesting an initial dissociation of the two dendrimers with additional loss of PN molecules occurring with increasing temperature. As observed in previous simulations (Figure 2E), the PN were partially dispersed inside the dendrimer rather than as the stable clusters observed with C16. Therefore, our results suggest that the supramolecular complexes formed in the experimental study ${ }^{20}$ of $\mathrm{C} 16$ dispersion by PAMAM resembled a multimolecular micelle structure, where the hydrocarbons in the core were stabilized by multiple dendrimers binding in the periphery. Such a multimolecular micelle structure of PAMAM was mainly the result of strong interligand interactions instead of liganddendrimer interactions (e.g., PN featured a stronger liganddendrimer interaction than $\mathrm{C} 16)$.

The ellipticity (Figure 5B) and $R g$ (Figure 5C) have also been computed to quantify the structural deviation of dendrimers binding to various ligands. In the case of $\mathrm{C} 16$, dendrimers bound to the cluster periphery and displayed notably larger $R g$ and ellipticity values compared to PN. As the temperature increased, higher thermal fluctuations resulted in increased $\mathrm{Rg}$ and symmetry (i.e., reduced ellipticity). As for PN, ligands were partitioned inside the dendrimers both in the multimolecular (at low temperatures) and in the unimolecular (at high temperatures) states, which resulted in more symmetrical structures with lower ellipticities than simulations with $\mathrm{C} 16$.

\section{CONCLUSION}

We applied DMD simulations to examine the molecular mechanisms of dendrimers as oil dispersants using two representative hydrocarbons, $\mathrm{C} 16$ and $\mathrm{PN}$. Our previous experimental study indicated that G4 PAMAM dendrimers dispersed linear and aromatic hydrocarbons quite differently, as evidenced in measurements of hydrodynamic size, ${ }^{20}$ loading capacity, and stability ${ }^{18}$ of the resulting complexes formed between the dendrimer and the hydrocarbons. On the basis of simulations of single dendrimers interacting with single and multiple hydrocarbons, we revealed a cooperativity in both $\mathrm{C} 16$ and PN dispersion by dendrimers. Such cooperativity arose from the increased hydrophobicity of the PAMAM interior upon ligand binding, which, in turn, facilitated binding of additional ligands. Such a cooperative binding of polyaromatic naphthalene with PAMAM has also been observed in recent allatom MD simulations with explicit solvent. ${ }^{42}$ Although PN displayed a stronger binding to PAMAM than $\mathrm{C} 16$, stronger interligand interactions led to a higher binding cooperativity for C16 to PAMAM than PN. Our simulations of multiple hydrocarbons interacting with each other suggest that the same strong interligand interaction between $\mathrm{C} 16$ molecules also contributes to their lower solubility than PN. In actual applications of PAMAM as dispersants, the targeted ligands are usually mixtures of different types of ligands. For example, in our previous experiments, ${ }^{18}$ the mixture of $\mathrm{C} 16$ with $8 \% \mathrm{PN}$ ligands was used as a "model crude", which displayed a higher binding cooperativity than PN. Although we did not model the mixture of $\mathrm{C} 16$ and $\mathrm{PN}$ in the current study, we expect that $\mathrm{C} 16$ will be a "good" solvent for PN and PAMAM can disperse the hydrocarbon mixtures in the same manner as $\mathrm{C} 16$ alone.

On the basis of the traditional unimolecular micelle paradigm of the PAMAM dendrimer, the binding of ligands is saturated once the dendrimer core is filled up and the maximum loading is reached. The unusually large size of molecular complexes formed by PAMAM dendrimers when dispersing C16 suggested a deviation from such an accepted scheme of PAMAM. By further simulating two dendrimers interacting with multiple hydrocarbons, we found that C16 and G4 
dendrimers formed a highly stable micelle structure across the simulated temperature range $(275-400 \mathrm{~K})$, in which a small C16 cluster was stabilized by multiple dendrimers on the periphery; the system of $\mathrm{PN}$ and dendrimers, however, consisted primarily of multiple $\mathrm{PN}$ molecules dispersed in the interior of single dendrimers. These results not only explain the phenomena observed in our earlier experimental studies ${ }^{18,20}$ but also illuminate the contrasting mechanisms of dendritic polymers for oil dispersion, where both ligand-ligand and ligand-dendrimer interactions contribute to conjure the dendrimer hosting capacity. Such dynamic capacity may serve as a basis for a range of dendrimer applications in environmental remediation, water purification, catalysis, and gene and drug delivery.

\section{ASSOCIATED CONTENT}

\section{S Supporting Information}

The Supporting Information is available free of charge on the ACS Publications website at DOI: 10.1021/acs.jpcc.5b04991.

The binding probability per ligand as a function of simulation temperatures, the radial density distributions of counterions and protonated primary amines, the PMF of one $\mathrm{C} 16$ binding with one PAMAM dendrimer as a function of the intermolecular distance; and a table listing the radius of gyration and ellipticity of G4PAMAM dendrimer (PDF)

\section{AUTHOR INFORMATION}

\section{Corresponding Author}

*E-mail: fding@clemson.edu.

\section{Notes}

The authors declare no competing financial interest.

\section{ACKNOWLEDGMENTS}

The work was supported in part by US EPA grant RD835182, NSF CBET-1232724, NIH R15ES022766-01A1, and Clemson University startup funds. The simulations were performed on the Palmetto high performance cluster, which is managed and maintained by Clemson University CCIT.

\section{REFERENCES}

(1) Helms, B.; Meijer, E. W. Dendrimers at Work. Science 2006, 313 (5789), 929-930.

(2) Esfand, R; Tomalia, D. A. Poly(amidoamine) (PAMAM) Dendrimers: From Biomimicry to Drug Delivery and Biomedical Applications. Drug Discovery Today 2001, 6 (8), 427-436.

(3) Svenson, S.; Tomalia, D. A. Dendrimers in Biomedical Applications-Reflections on the Field. Adv. Drug Delivery Rev. 2005, 57 (15), 2106-2129.

(4) Tian, W.-d.; Ma, Y.-q. Theoretical and Computational Studies of Dendrimers as Delivery Vectors. Chem. Soc. Rev. 2013, 42 (2), 705727.

(5) Naylor, A. M.; Goddard, W. A.; Kiefer, G. E.; Tomalia, D. A. Starburst Dendrimers. 5. Molecular Shape Control. J. Am. Chem. Soc. 1989, 111 (6), 2339-2341.

(6) Newkome, G. R.; Moorefield, C. N.; Baker, G. R.; Johnson, A. L.; Behera, R. K. Alkane Cascade Polymers Possessing Micellar Topology: Micellanoic Acid Derivatives. Angew. Chem., Int. Ed. Engl. 1991, 30 (9), $1176-1178$

(7) Watkins, D. M.; Sayed-Sweet, Y.; Klimash, J. W.; Turro, N. J.; Tomalia, D. A. Dendrimers with Hydrophobic Cores and the Formation of Supramolecular Dendrimer-Surfactant Assemblies. Langmuir 1997, 13 (12), 3136-3141.
(8) Moreno-Bondi, M. C.; Orellana, G.; Turro, N. J.; Tomalia, D. A. Photoinduced Electron-Transfer Reactions to Probe the Structure of Starburst Dendrimers. Macromolecules 1990, 23 (3), 910-912.

(9) Turro, N. J.; Barton, J. K.; Tomalia, D. A. Molecular Recognition and Chemistry in Restricted Reaction Spaces. Photophysics and Photoinduced Electron Transfer on the Surfaces of Micelles, Dendrimers, and DNA. Acc. Chem. Res. 1991, 24 (11), 332-340.

(10) Gopidas, K. R.; Leheny, A. R.; Caminati, G.; Turro, N. J.; Tomalia, D. A. Photophysical Investigation of Similarities between Starburst Dendrimers and Anionic Micelles. J. Am. Chem. Soc. 1991, 113 (19), 7335-7342.

(11) Maiti, P. K.; Bagchi, B. Diffusion of Flexible, Charged, Nanoscopic Molecules in Solution: Size and $\mathrm{pH}$ Dependence for PAMAM Dendrimer. J. Chem. Phys. 2009, 131 (21), 214901.

(12) Crampton, H. L.; Simanek, E. E. Dendrimers as Drug Delivery Vehicles: Non-Covalent Interactions of Bioactive Compounds with Dendrimers. Polym. Int. 2007, 56 (4), 489-496.

(13) Yiyun, C.; Tongwen, X. Dendrimers as Potential Drug Carriers. Part I. Solubilization of Non-Steroidal Anti-Inflammatory Drugs in the Presence of Polyamidoamine Dendrimers. Eur. J. Med. Chem. 2005, 40 (11), 1188-1192.

(14) Asthana, A.; Chauhan, A. S.; Diwan, P. V.; Jain, N. K. Poly(amidoamine) (PAMAM) Dendritic Nanostructures for Controlled Sitespecific Delivery of Acidic Anti-Inflammatory Active Ingredient. AAPS PharmSciTech 2005, 6 (3), E536-E542.

(15) Kolhe, P.; Misra, E.; Kannan, R. M.; Kannan, S.; Lieh-Lai, M. Drug Complexation, in Vitro Release and Cellular Entry of Dendrimers and Hyperbranched Polymers. Int. J. Pharm. 2003, 259 (1-2), 143-160.

(16) Lin, S.-T.; Maiti, P. K.; Goddard, W. A. Dynamics and Thermodynamics of Water in PAMAM Dendrimers at Subnanosecond Time Scales. J. Phys. Chem. B 2005, 109 (18), 8663-8672.

(17) Liu, Y.; Porcar, L.; Hong, K.; Shew, C.-Y.; Li, X.; Liu, E.; Butler, P. D.; Herwig, K. W.; Smith, G. S.; Chen, W.-R. Effect of Counterion Valence on the $\mathrm{pH}$ Responsiveness of Polyamidoamine Dendrimer Structure. J. Chem. Phys. 2010, 132 (12), 124901.

(18) Geitner, N. K.; Wang, B.; Andorfer, R. E.; Ladner, D. A.; Ke, P. C.; Ding, F. Structure-Function Relationship of PAMAM Dendrimers as Robust Oil Dispersants. Environ. Sci. Technol. 2014, 48 (21), $12868-12875$.

(19) Maiti, P. K.; Çağın, T.; Wang, G.; Goddard, W. A. Structure of PAMAM Dendrimers: Generations 1 through 11. Macromolecules 2004, 37 (16), 6236-6254.

(20) Geitner, N. K.; Bhattacharya, P.; Steele, M.; Chen, R.; Ladner, D. A.; Ke, P. C. Understanding Dendritic Polymer-hydrocarbon Interactions for Oil Dispersion. RSC Adv. 2012, 2 (25), 9371-9375.

(21) Bhattacharya, P.; Conroy, N.; Rao, A. M.; Powell, B. A.; Ladner, D. A.; Ke, P. C. PAMAM Dendrimer for Mitigating Humic Foulant. RSC Adv. 2012, 2 (21), 7997-8001.

(22) Diallo, M. S.; Balogh, L.; Shafagati, A.; Johnson, J. H., Jr.; Goddard, W. A., III; Tomalia, D. A. Poly(amidoamine) Dendrimers: A New Class of High Capacity Chelating Agents for $\mathrm{Cu}(\mathrm{II})$ Ions. Environ. Sci. Technol. 1999, 33 (5), 820-824.

(23) National Research Council (U.S.) Committee on Effectiveness of Oil Spill Dispersants. Using Oil Spill Dispersants on the Sea; National Academy Press: Washington, DC, 1989; p 352.

(24) Hemmer, M. J.; Barron, M. G.; Greene, R. M. Comparative Toxicity of Eight Oil Dispersants, Louisiana Sweet Crude Oil (LSC), and Chemically Dispersed LSC to Two Aquatic Test Species. Environ. Toxicol. Chem. 2011, 30 (10), 2244-2252.

(25) Anderson, S. E.; Franko, J.; Lukomska, E.; Meade, B. J. Potential Immunotoxicological Health Effects Following Exposure to COREXIT 9500A during Cleanup of the Deepwater Horizon Oil Spill. J. Toxicol. Environ. Health, Part A 2011, 74 (21), 1419-1430.

(26) Goodbody-Gringley, G.; Wetzel, D. L.; Gillon, D.; Pulster, E.; Miller, A.; Ritchie, K. B. Toxicity of Deepwater Horizon Source Oil and the Chemical Dispersant, Corexit ${ }^{\circledR}$ 9500, to Coral Larvae. PLoS One 2013, 8 (1), e45574. 
(27) Geitner, N. K.; Powell, R. R.; Bruce, T.; Ladner, D. A.; Ke, P. C. Effects of Dendrimer Oil Dispersants on Dictyostelium Discoideum. RSC Adv. 2013, 3 (48), 25930-25936.

(28) Mandal, T.; Dasgupta, C.; Maiti, P. K. Nature of the Effective Interaction between Dendrimers. J. Chem. Phys. 2014, 141 (14), 144901.

(29) Rapaport, D. C. The Art of Molecular Dynamics Simulation, 2nd ed.; Cambridge University Press: New York, 2004.

(30) Allen, M. P.; Tildesley, D. J. Computer Simulation of Liquids; Clarendon Press: New York, 1989.

(31) Ding, F.; Tsao, D.; Nie, H.; Dokholyan, N. V. Ab Initio Folding of Proteins with All-Atom Discrete Molecular Dynamics. Structure 2008, 16 (7), 1010-1018.

(32) Dagliyan, O.; Proctor, E. A.; D'Auria, K. M.; Ding, F.; Dokholyan, N. V. Structural and Dynamic Determinants of ProteinPeptide Recognition. Structure 2011, 19 (12), 1837-1845.

(33) Ding, F.; Furukawa, Y.; Nukina, N.; Dokholyan, N. V. Local Unfolding of $\mathrm{Cu}, \mathrm{Zn}$ Superoxide Dismutase Monomer Determines the Morphology of Fibrillar Aggregates. J. Mol. Biol. 2012, 421 (4-5), $548-560$.

(34) Yin, S.; Biedermannova, L.; Vondrasek, J.; Dokholyan, N. V. MedusaScore: An Accurate Force Field-Based Scoring Function for Virtual Drug Screening. J. Chem. Inf. Model. 2008, 48 (8), 1656-1662.

(35) Ding, F.; Dokholyan, N. V. Emergence of Protein Fold Families through Rational Design. PLoS Comput. Biol. 2006, 2 (7), e85.

(36) Neria, E.; Fischer, S.; Karplus, M. Simulation of Activation Free Energies in Molecular Systems. J. Chem. Phys. 1996, 105, 1902.

(37) Lazaridis, T.; Karplus, M. Effective Energy Functions for Protein Structure Prediction. Curr. Opin. Struct. Biol. 2000, 10, 139-145.

(38) Manning, G. S. Limiting Laws and Counterion Condensation in Polyelectrolyte Solutions I. Colligative Properties. J. Chem. Phys. 1969, 51 (3), 924-933.

(39) Kumar, S.; Rosenberg, J. M.; Bouzida, D.; Swendsen, R. H.; Kollman, P. A. THE Weighted Histogram Analysis Method for FreeEnergy Calculations on Biomolecules. I. The Method. J. Comput. Chem. 1992, 13 (8), 1011-1021.

(40) Neria, E.; Fischer, S.; Karplus, M. Simulation of Activation Free Energies in Molecular Systems. J. Chem. Phys. 1996, 105 (5), 19021921.

(41) Maingi, V.; Kumar, M. V. S.; Maiti, P. K. PAMAM DendrimerDrug Interactions: Effect of $\mathrm{pH}$ on the Binding and Release Pattern. J. Phys. Chem. B 2012, 116 (14), 4370-4376.

(42) DeFever, R. S.; Geitner, N. K.; Bhattacharya, P.; Ding, F.; Ke, P. C.; Sarupria, S. PAMAM Dendrimers and Graphene: Materials for Removing Aromatic Contaminants from Water. Environ. Sci. Technol. 2015, 49 (7), 4490-4497. 


\section{Deviation From the Unimolecular Micelle Paradigm of PAMAM Dendrimers Induced by Strong Inter- Ligand Interactions}

Bo Wang, ${ }^{\dagger}$ Nicholas K Geitner, \# Thomas P Davis,,$\$$ Pu Chun Ke, ${ }^{\S}$ David A Ladner, $\neq$ and Feng Dingt $^{*}$

† Department of Physics and Astronomy, Clemson University, Clemson, SC 29634

\# Department of Civil and Environmental Engineering, Duke University, NC 27708

$\S$ ARC Centre of Excellence in Convergent Bio-Nano Science and Technology, Monash Institute of Pharmaceutical Sciences, Monash University, 381 Royal Parade, Parkville, VIC 3052, Australia

ฯ Department of Chemistry, Warwick University, Gibbet Hill, Coventry, CV4 7AL, United Kingdom

‡ Department of Environmental Engineering and Earth Sciences, Clemson University, Clemson, SC 29634

\section{Supporting Information}

Three supporting figures

One supporting tables 


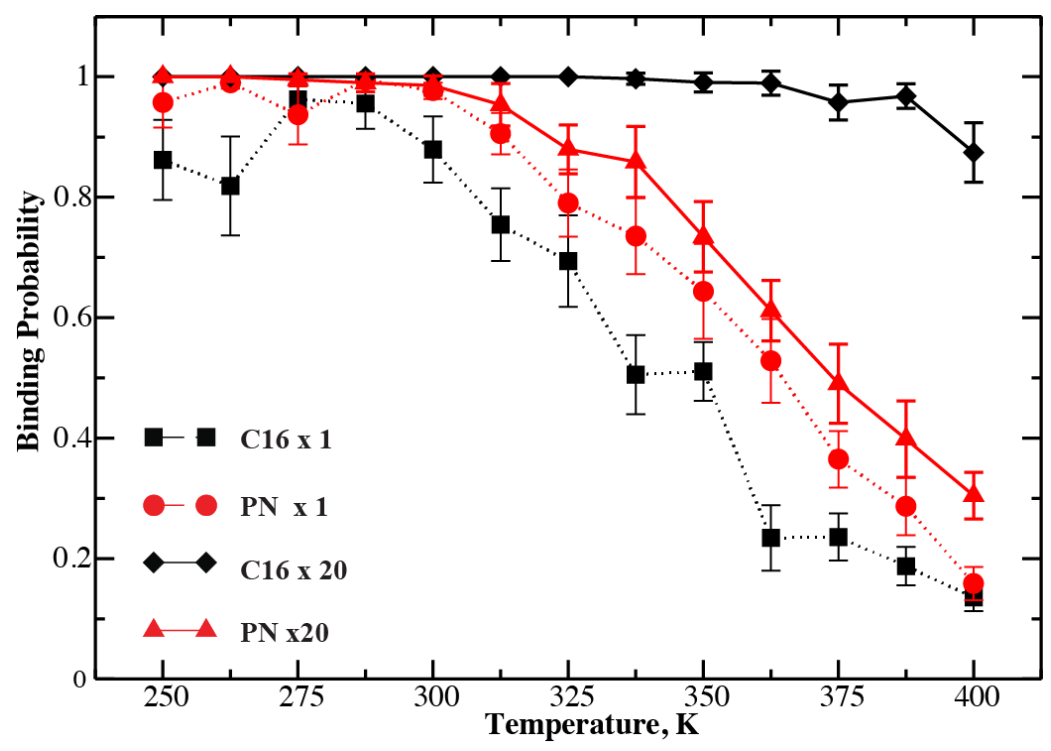

Figure S1. The binding probability per ligand, $P_{b i n d}$, was obtained by normalizing with the total number of ligands for simulations of multiple ligands (x20) binding PAMAM (Fig. 2B). For comparison, $P_{b i n d}$, for single hydrocarbon binding simulations was also included (dot lines). 


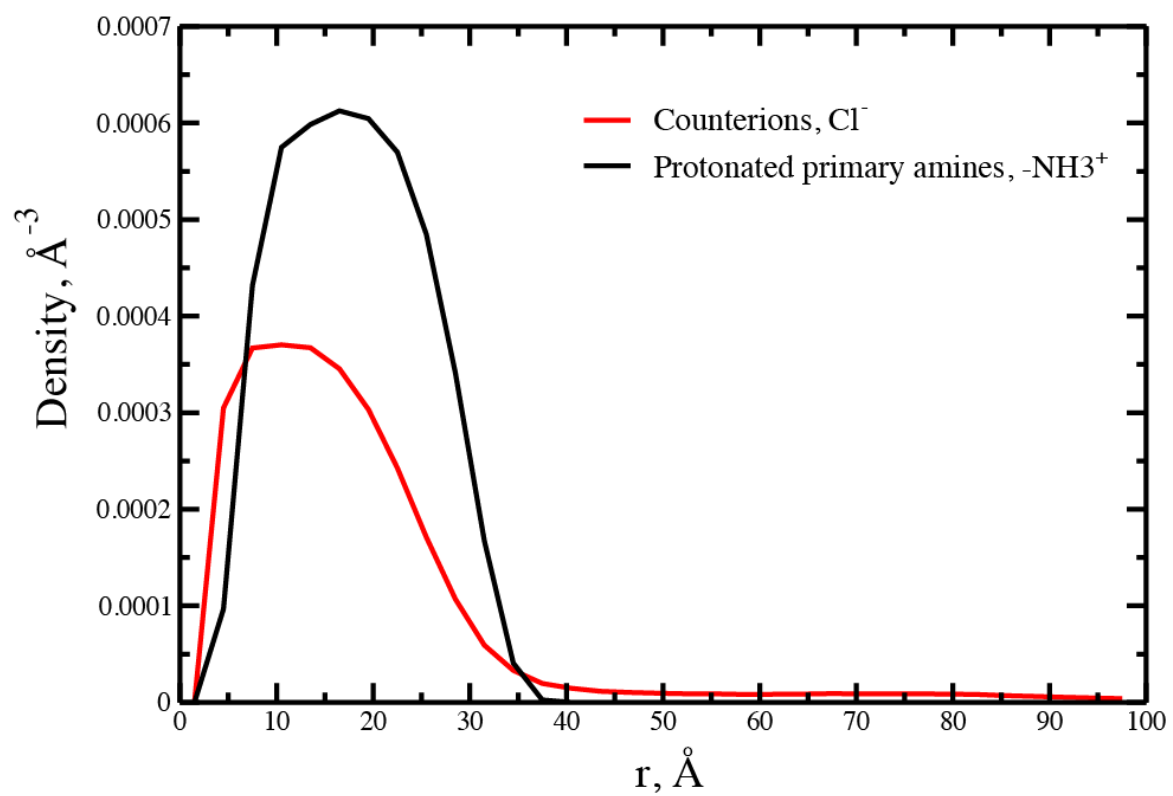

Figure S2. The radial density distributions of counter ions, $\mathrm{Cl}^{-}$, and protonated primary amines, $-\mathrm{NH}_{3}{ }^{+}$. The center of the PAMAM core was used as the reference point, and the density was computed as the number of atoms per unit volume with a distance bin size of $2.5 \AA$ A. The distributions were averaged over time when the molecular systems reached equilibrium. 


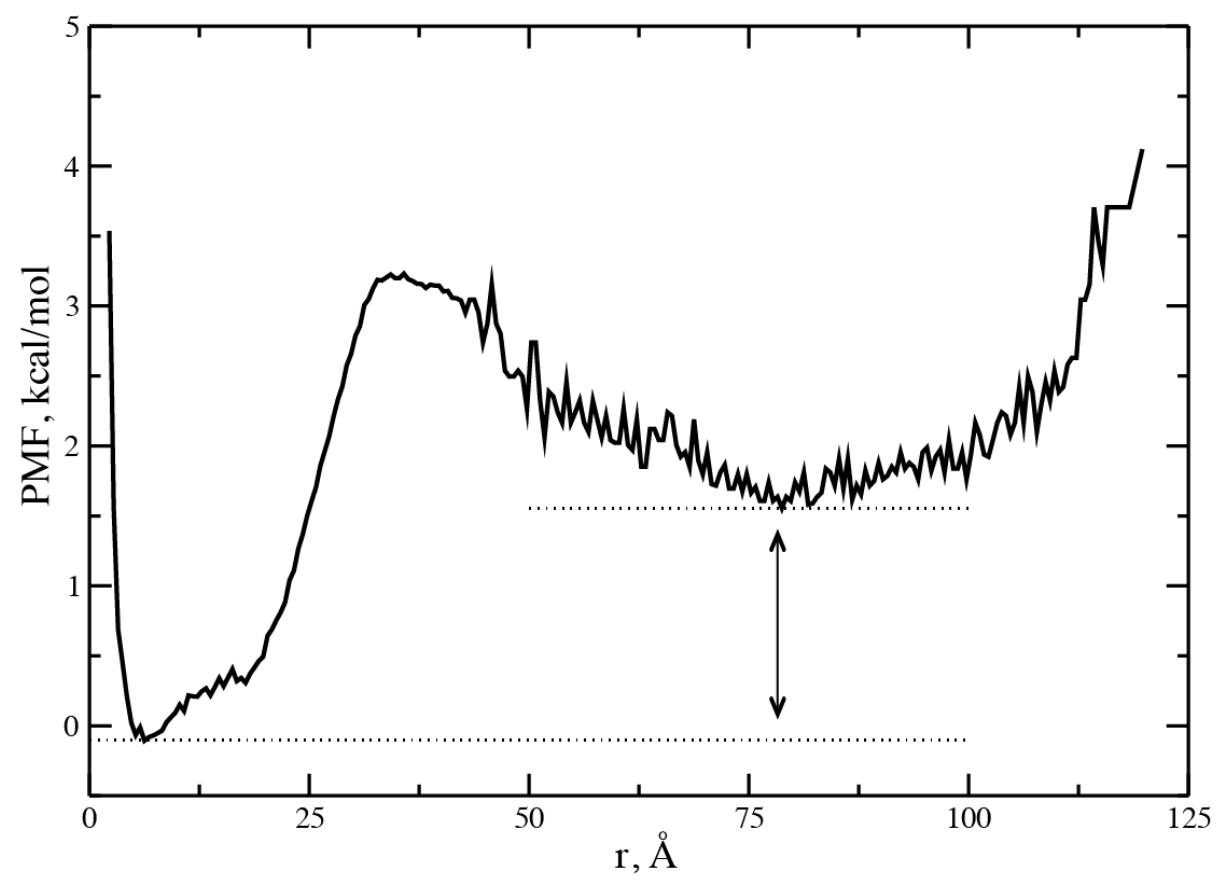

Figure S3. The potential of mean force (PMF) of one C16 binding with one PAMAM dendrimer as the function of the inter-molecular distance, $\boldsymbol{r}$, at $\mathrm{T}=300 \mathrm{~K}$. The results were calculated from umbrella sampling simulations using the WHAM method (Methods). The PMF minimum at the short $\boldsymbol{r}$ corresponds to the bound state with a strong inter-molecular interaction. As $r$ increases beyond the free energy barrier around $\sim 30 \AA$ (corresponding to the termini of the dendrimer as calculated in Fig. S2), a decrease of PMF occurs, resulting from increased translational degrees of freedom with the minimum around $\sim 80 \AA$, half of the simulation box size. Dot lines indicate the lowest free energy of bounded and unbounded states. 
Table S1. Radius of gyration and ellipiticity of G4-PAMAM dendrimer. The DMD simulation was performed at $\mathrm{T}=300 \mathrm{~K}$ for $50 \mathrm{~ns}$.

\begin{tabular}{|l|l|l|}
\hline \multirow{2}{*}{ Radius of gyration, $\AA$} & Liu et al. $^{1}$ - SANS & This work \\
\cline { 2 - 3 } & $21.4(0.4)$ & $20.2(0.6)$ \\
\hline \multirow{2}{*}{ Ellipticity } & Maiti et al. ${ }^{2}$ - MD & This work \\
\cline { 2 - 3 } & $0.70(0.04)$ & $0.73(0.07)$ \\
\hline
\end{tabular}

\section{REFERENCES}

(1) Liu, Y.; Porcar, L.; Hong, K.; Shew, C.-Y.; Li, X.; Liu, E.; Butler, P. D.; Herwig, K. W.; Smith, G. S.; Chen, W.-R. Effect of Counterion Valence on the pH Responsiveness of Polyamidoamine Dendrimer Structure. J. Chem. Phys. 2010, 132 (12), 124901.

(2) Maiti, P. K.; Çağın, T.; Wang, G.; Goddard, W. A. Structure of PAMAM Dendrimers: Generations 1 through 11. Macromolecules 2004, 37 (16), 6236-6254. 